\title{
Personalized Pulmonary Trunk Modeling for Intervention Planning and Valve Assessment Estimated from CT Data
}

\author{
Dime Vitanovski ${ }^{2,3}$, Razvan Ioan Ionasec ${ }^{1,5, \star}$, Bogdan Georgescu $^{1}$, \\ Martin Huber ${ }^{2}$, Andrew Mayall Taylor ${ }^{4}$, Joachim Hornegger ${ }^{3}$, \\ and Dorin Comaniciu ${ }^{1}$
}

1 Integrated Data Systems, Siemens Corporate Research, Princeton, USA

2 Software and Engineering, Siemens Corporate Technology, Erlangen, Germany

3 Chair of Pattern Recognition, Friedrich-Alexander-University, Erlangen, Germany

${ }^{4}$ UCL Institute of Child Health \& Great Ormond Street Hospital for Children

${ }^{5}$ Computer Aided Medical Procedures, Technical University Munich, Germany

razvan.ionasec@siemens.com

\begin{abstract}
Pulmonary valve disease affects a significant portion of the global population and often occurs in conjunction with other heart dysfunctions. Emerging interventional methods enable percutaneous pulmonary valve implantation, which constitute an alternative to open heart surgery. As minimal invasive procedures become common practice, imaging and non-invasive assessment techniques turn into key clinical tools. In this paper, we propose a novel approach for intervention planning as well as morphological and functional quantification of the pulmonary trunk and valve. An abstraction of the anatomic structures is represented through a four-dimensional, physiological model able to capture large pathological variation. A hierarchical estimation, based on robust learning methods, is applied to identify the patient-specific model parameters from volumetric CT scans. The algorithm involves detection of piecewise affine parameters, fast centre-line computation and local surface delineation. The estimated personalized model enables for efficient and precise quantification of function and morphology. This ability may have impact on the assessment and surgical interventions of the pulmonary valve and trunk. Experiments performed on 50 cardiac computer tomography sequences demonstrated the average speed of 202 seconds and accuracy of $2.2 \mathrm{~mm}$ for the proposed approach. An initial clinical validation yielded a significant correlation between model-based and expert measurements. To the best of our knowledge this is the first dynamic model of the pulmonary trunk and right ventricle outflow track estimated from CT data.
\end{abstract}

\section{Introduction}

Valvular heart disease (VHD) is an important cardiac disorder that affects a large number of patients and often requires operative intervention. In most cases, pulmonary abnormality occurs in conjunction with other heart diseases and can

\footnotetext{
^ Corresponding author.
} 
be caused by congenital defects and pulmonary hypertension endocarditis. Such conditions require constant monitoring and a complex clinical workflow which includes: patient evaluation, percutaneous intervention planning, valve replacement and repair and follow-up studies.

Until recently, the pulmonary valve replacement has been performed surgically on open heart 1, with all associated risks: high morbidity, incidence of neurological damage, stroke and repeated valve replacement 2. Minimal invasive procedures for the pulmonary valve are less traumatic, reducing valve replacement risk [3. Percutaneous pulmonary valve implantation (PPVI) 4] is a new developed technique for transcatheter placement of a valve stent. The main difficulties of PPVI are: the assessment of the pulmonary trunk and the right ventricle outflow track (RVOT $<22 \mathrm{~mm}$ ) before treatment 4 , the classification of patients suitable for the procedure [5] and identification of the exact location for anchoring the stent [6]. Hence, precise assessment of the morphology and dynamics is crucial for the pre-procedural planning and successful intervention of PPVI.

Cardiac CT imaging is often performed when high spatial resolution, soft tissue contrast or dynamics is essential. The key advantage is the ability to acquire multiple non-invasive and accurate scans required for evaluation. In standard clinical settings these are the imaging techniques used to gain the information about the shape of the RVOT and the pulmonary artery. The acquired data (4D $\mathrm{CT}$ ) is usually translated into sets of $2 \mathrm{D}$ planes for manual quantification and visual evaluation due to the lack of appropriate methods and tools for processing 3D/4D information. Measurements are tedious to obtain and moreover known to be affected by inaccuracies, as $2 \mathrm{D}$ alignment and sectioning is ambiguous and might lead to misinterpretation and distensibility [4].

A dynamic personalized model is expected to enhance quantification accuracy and non-invasive visualization of the human pulmonary trunk and RVOT. Recently, Schievano et. al. [5] proposed a patient specific 3D model manual extracted from MRI data. Although it may positively impact patient evaluation and procedure planning, the modeling requires a long time, the accuracy is influenced by user experience, and does not include dynamics.

Within this paper we present a dynamic model of the pulmonary trunk automatically estimated from CT data. The segmentation task is performed by fitting a 4D generic model to the patient specific image data. The proposed workflow, from modeling to quantification, involves three stages. In the first place, a generic model of the pulmonary artery is computed from a comprehensive training set (see Section 2). This is fitted to the available volumetric scans using robust machine-learning methods in order to obtain a patient specific physiological model (Section 3). Finally, the morphology and function of the artery is efficiently quantified from the personalized abstract representation (Section 4.1).

Extensive experiments are performed on a data set of $50 \mathrm{CT}$ patients (503 Volumes), which includes a diverse range of morphological and pathological variation and patients' age. Results of the experiments demonstrated the average 
accuracy of $2.2 \mathrm{~mm}$ and speed of 202 seconds for the proposed approach on cardiac CT data (Section 4.2). An initial clinical validation performed demonstrates a significant correlation between model-based and manually performed expert measurements.

\section{Model Representation}

In order to accurately represent morphology and dynamics, our model design is consistent with the anatomy and physiology of the pulmonary trunk. The architecture of the model is anatomically oriented and includes all clinical relevant structures. Geometries are represented parametrically using non-uniform rational B-Splines (NURBS) [7], which is standard mathematical formulation in computation modeling.

Initially, the pulmonary trunk is represented by five anatomical landmarks: Trigone $\left(L_{t}\right)$, RVOT $\left(L_{\text {rvot }}\right)$, Main-Bifurcation $\left(L_{m b}\right)$, Left-Bifurcation $\left(L_{l b}\right)$ and Right-Bifurcation $\left(L_{r b}\right)$. The Trigone defines the splitting point between the right ventricle outflow track and the tricuspid valve plane. The RVOT landmark is located on the same plane as the Trigone, parallel to the tricuspid valve plane. Main-Bifurcation defines the center of the trunk branching, while LeftBifurcation and Right-Bifurcation mark the branches' end points (see Fig. 1(a)).

Constrained by the landmarks, the artery's center lines form the second abstraction layer (see Fig. 1(b)). The Main Center Line is bounded by the RVOT and Bifurcation. The Left and the Right Center Lines extend from the main bifurcation landmark to the Left-Bifurcation and Right-Bifurcation landmark, respectively.

The main pulmonary artery along with its bifurcations is modeled as parametric closed surface similar to a deformed cylinder. NURBS are used as the parametric representation due to the compact mathematical formulation and convenient properties. The topology and morphology of the arterial structure is characterized and constrained by the previously defined landmarks and centerlines. In order to obtain the physiological model of the pulmonary artery, a cylindrical structure along with the center line has been computed (see Fig. 1(c)).

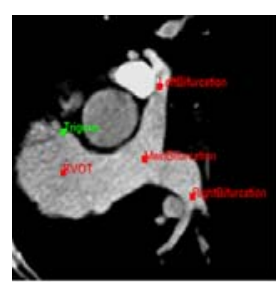

a)

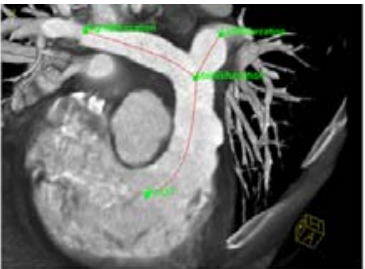

b)

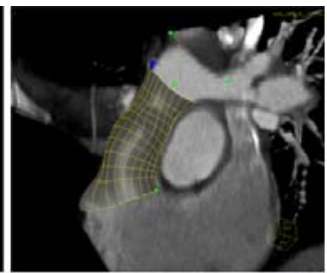

c)

Fig. 1. Model representation 


\section{Model Estimation}

The abstract parameters of the pulmonary trunk proposed in Section 2 are determined for a specific patient from the available data (four dimensional CT). A specific instance of the model is constrained in four-dimensional Euclidean space by 5 landmarks, 3 centerlines (determined by 27 discrete points) and 3 surfaces (determined by 966 control points) cumulating into $5^{*} \mathrm{~T}^{1}+3^{*} \mathrm{~T}^{*}(9+$ 322 ) parameters. The high dimensionality of the parameter space makes the direct estimation in the original space a very difficult task. Therefore, we proposed a four-step approach by first detecting a piecewise similarity transformation, followed by landmark, center line and full dynamic model detection.

\subsection{Similarity Transformation and Landmark Detection}

For each landmark except the triagone, which is included into the RVOT box $\left.\theta_{\text {rvot }}\right)$, we define a corresponding bounding box, parameterized by an affine transformation $\theta=\left(X, Y, Z, \alpha, \beta, \chi, S_{x}, S_{y}, S_{z}\right)$. Each box characterizes one anatomical structure: RVOT plane is defined by $\theta_{\text {rvot }}$ with orientation given by the center line's tangent, $\theta_{m b}$ defines the main bifurcation plane with orientation orthogonal to the trunk, respectively $\theta_{l b}$ and $\theta_{r b}$ for the left and right bifurcation plane.

As the object localization task is formulated as a classification problem, a robust detector which uses 3D Haar and Steerable Features [8] is trained using a PBT (Probabilistic Boosting Tree) [9]. In order to efficiently perform learning in high dimension space, we marginalize the search space by means of MSL [10] into subspaces which are gradually increased. Consequently, the parameter estimation problem is splitted into three steps where classifiers, parameterized by the current subset of the affine values, are sequentially trained on the subspaces: position, position-rotation and full affine transformation. A further speed improvement is achieved by using a pyramidal-based coarse-to-fine approach and searching in low-resolution $(3 \mathrm{~mm})$ volume.

The estimated parameters $\theta_{i}$ initialize the landmarks $\left(L_{t}, L_{r v o t}, L_{m b}, L_{l b}, L_{r b}\right)$ by its position and orientation and constrain the search domain $D_{i}$ by its scale. A further accuracy improvement is achieved by learning a discriminative 2 Level PBT classifier $\boldsymbol{H}_{L}$ which learns the target distribution

$$
p\left(\boldsymbol{L}_{i} \mid x_{l}, y_{l}, z_{l}, \boldsymbol{I}\right)=\boldsymbol{H}_{i}\left(x_{l}, y_{l}, z_{l} \mid \boldsymbol{I}\right),\left(x_{l}, y_{l}, z_{l}\right) \in D_{i}, i=1 . .5
$$

in $1 \mathrm{~mm}$ volume resolution, where $p\left(L_{i} \mid x_{l}, y_{l}, z_{l}, I\right)$ is the probability of the presence of $\mathbf{L}_{i}$ at location $\left(x_{l}, y_{l}, z_{l}\right)$.

\subsection{Center Line Detection}

In this step we initialize the center lines by previously detected landmarks. The main center line which goes through the center of the pulmonary trunk

${ }^{1} \mathrm{~T}$ represents discrete time steps (10 for a regular $4 \mathrm{D}$ cardiac $\mathrm{CT}$ acquisitions). 
is bounded by the $L_{\text {rvot }}$ and $L_{m b}$, whereas the left and right center lines are constrained by the $L_{l b}$ and $L_{r b}$, respectively $L_{m b}$ (see Section 2).

Automatic refinement of the center-line curves is achieved by applying a robust circle detector, which is trained using the PBT algorithm and Haar features. In the learning phase positive and negative samples are generated in circular form, following the annotated curves. In the detection stage, an incremental approach is used for searching $3 \mathrm{D}$ center points $Q_{i}$ on a series of parallel planes. These are bounded by the corresponding landmarks, while their normal is given by the initial center-line and updated after each detected center point.

A least-square approach is used to fit a parametric NURBS curve $C$ to the discrete set of detected center points $Q_{i}$. The LSE problem is solved by minimizing an objective function (EqL) with respect to the control points $P^{k}$

$$
E=\sum_{i=1}^{N-1}\left|Q_{i}-C\left(\bar{u}_{i}\right)\right|^{2}, \underbrace{C(u)}_{u \in[0,1]}=\frac{\sum_{k=0}^{n} N_{k, d}(u) w_{k} \boldsymbol{P}^{k}}{\sum_{k=0}^{n} N_{k, d}(u) w_{k}}
$$

\subsection{Dynamic Model Detection}

The full surface model is initialized by projecting a mean shape, averaged over the whole annotations from the training set, into the patient specific anatomy. The projection is achieved using a piecewise affine transformation defined from the detected landmarks and center-lines.

A set of center-points $C_{i}$ are equidistantly sampled along the detected centerlines. At each location, we construct a local coordinate system, using the centerline's tangent and projected RVOT-Triagone to define the orientation. The control points $P^{k}$ are associated to one of the local coordinate systems, based on their Euclidean distance to the local origin. Using a coordinate system transformation we map the mean-shape control points $P^{k}$ to the corresponding image location (see Fig. 2).

The transformed mean model provides accurate global fitting into the patient's specific anatomy. However, further local processing for precise object delineation is required. A boundary detector, trained by PBT in combination with steerable features, tests a set of hypotheses along the surface normal and moves the shape towards position with highest boundary probability. Spatially smooth
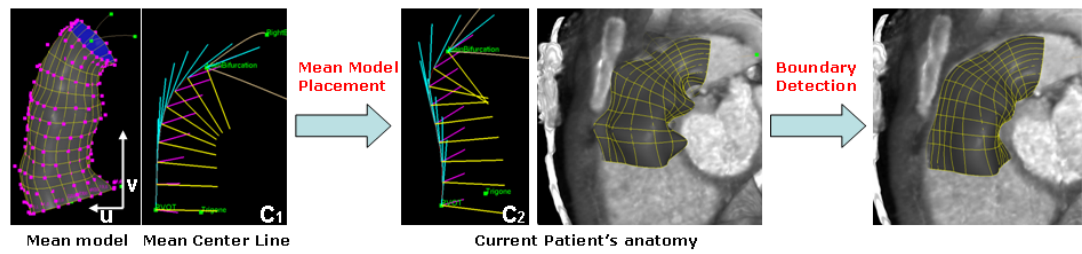

Fig. 2. Piecewise mean model placement 
surface is obtained by projecting the detected surface into the corresponding shape subspace [1].

Temporal consistency of the dynamic model is enhanced similar as in 12 . One-step-forward prediction approach is applied to estimate the motion prior using motion manifold learning, which gives an appropriate initialisation for the boundary detectors. Optical flow is fused with tracking by detection to achieve temporal consistency.

\section{Results}

\subsection{Results on Pulmonary Trunk Model Estimation}

The performance of the proposed approaches is evaluated on $504 \mathrm{D}$ CT (503 volumes) studies, associate with a manual annotation considered to be the ground truth. Included data of patients with various ages (from 5 months infant to 65 years old adult) lead to significant differences in morphology and pathology of the pulmonary trunk. CT data is acquired using different protocols, resulting in volume sizes between $153 \times 153 \times 80$ and $512 \times 512 \times 350$ and voxel resolutions from $0.28 \mathrm{~mm}$ to $2.0 \mathrm{~mm}$.

The accuracy of our detection framework is evaluated by using a three-fold cross validation. Table 1 summarizes the results from the cross-validation for the full model fitting. The detection error for the landmarks and the bounding box is measured from the Euclidean distance between the ground truth and detected landmarks, respectively bounding box corners. The point-to-mesh and pointto-curve measurement error was used for the boundary and the center lines detectors, respectively. As expected, the error constantly decreases with each estimation stage. We obtain an average precision of $2.2 \mathrm{~mm}$ and computation time of $202 \mathrm{sec}$ on a standard $2.0 \mathrm{GHz}$ Dual Core PC.

\subsection{Results on Clinical Evaluation}

The accuracy of the automatic quantification of the pulmonary trunk is demonstrated by comparing a set of morphological and dynamic based measurements derived from the model to expert measurements and literature reported values.

Schievano et.al. [5] has proposed a set of measurements: max (RVOT) diameter, min diameter and diameter at the main bifurcation region from which the exact location for anchoring the valve stent is defined [6]. PPVI intervention

Table 1. Detection accuracy

\begin{tabular}{|c|c|c|c|}
\hline & Mean Error(mm) & Median $(\mathrm{mm})$ & Std.Dev $(\mathrm{mm})$ \\
\hline Bounding box & 6.5 & 7.1 & 2.7 \\
\hline Landmarks & 3.9 & 4.5 & 2.1 \\
\hline Center Line & 4.5 & 4.6 & 1.9 \\
\hline Boundary & 2.2 & 1.1 & 2.1 \\
\hline
\end{tabular}


Table 2. Model-based quantification error with respect to literature(upper table) and expert measurements(lower table)

\begin{tabular}{|l|c|c|}
\hline$(\mathrm{mm})$ & Observed & Literature \\
\hline Bifurcation Diameter & $30.2 \pm 1.6$ & $30.7 \pm 3.6$ \\
\hline$(\mathrm{mm})$ & Mean Err. & Std. Dev. \\
\hline Min Diameter & 1.99 & 0.64 \\
\hline Max Diameter & 4.06 & 2.09 \\
\hline Bifurcation Diameter & 1.04 & 0.65 \\
\hline
\end{tabular}

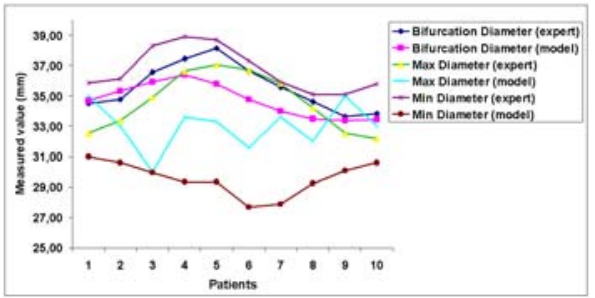

is avoided by patients with RVOT diameter $>22 \mathrm{~mm}$ due to device limitation 1145. Hence, the accuracy of the measurements is crucial for the success of the PPVI. The evaluation results of the proposed model-based quantification method are compared with results from the literature [13] and from experts (see Table 2).

\subsection{Results on Model Based Patient Selection Suitable for PPVI}

The morphology of the pulmonary trunk is a major determinant of suitability for PPVI [5]. Intervention in unsuitable patients exposes patients to unnecessary invasive catherization. A valve stent placed in such patients has a high probability of proximal device dislodgment. Schievano et.al. [5] proposed the classification of various morphologies in five groups: pyramidal shape (type I), constant diameter (type II), inverted pyramidal shape (type III), wide centrally but narrowed proximally and distally (type IV), and narrowed centrally but wide proximally and distally (type V). Patients from type I are considered to be unsuitable for PPVI due to the narrow artery and high probability of device migration. Hence, the main challenge lies in discriminating anatomies of type I from other classes.

Similar as in [14] we propose a robust shape-based patient selection for PPVI. Shape features extracted from the estimated pulmonary trunk (see Section 2) are used to learn a discriminative distance function using the Random Forest

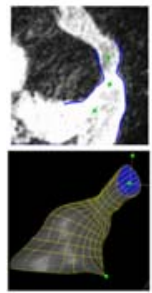

Type 1

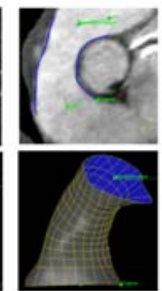

Type 2

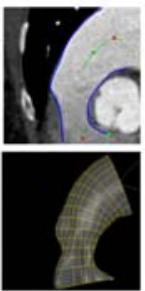

Type 3

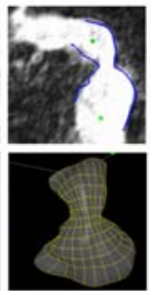

Type 4

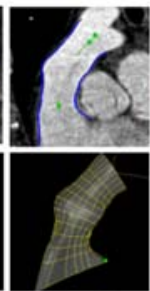

Type 5

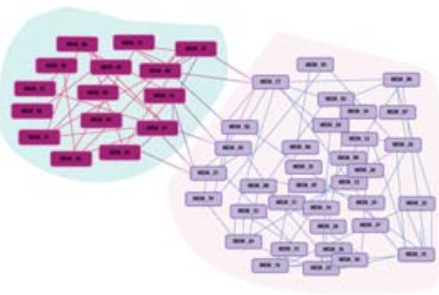

Fig. 3. Left: examples of 3D models for each morphological type. Right: Model-based patient classification: patient unsuitable (light cyan cluster) / suitable (light violet cluster) for PPVI intervention. 
in the product space. This is applied to classify subjects into two classes: PPVI suitable and PPVI unsuitable.

The results of our method are illustrated in Fig. 3. The accuracy of the model based classification is validated by leave-one-out cross-validation. We observed correct classification in $91 \%$ of the $\mathbf{5 0}$ cases. The proposed method has the potential to significantly improve accuracy and reproducibility of patient selection for PPVI.

\section{Discussion}

This paper proposes a novel dynamic model for morphological and functional quantification of the pulmonary trunk and RVOT for PPVI intervention. Integrating all the relevant data obtained during a CT scan in a holistic fashion, the physiology of the patient's anatomy is accurately represented. An efficient and robust learning-based algorithm was proposed to compute a patient specific pulmonary model from the available image data (CT). The estimated model can be utilized to extract morphological information, direct measurements of the pulmonary trunk and the pulmonary dynamics over the cardiac cycle. Automatic model-based dynamic measurements improve the patient evaluation and selection as well as the planning of the interventional procedure. Shape-based morphology type assessment in combination with RVOT size computation provides precise selection of proper morphology appropriate for percutaneous implantable pulmonary valves and avoids unnecessary patient catheterization.

\section{References}

1. Boudjemline, Y., Agnoletti, G., Bonnet, D., Sidi, D., Bonhoeffer, P.: Percutaneous pulmonary valve replacement in a large right ventricular outflow tract: An experimental study. American College of Cardiology 43, 1082-1087 (2004)

2. Parr, J., Kirklin, J., Blackstone, E.: The early risk of re-replacement of aortic valves. The Annals of Thoracic Surgery 23(4), 319-322 (1977)

3. Carnaghan, H.: Percutaneous pulmonary valve implantation and the future of replacement. Science and Technology 20(1), 319-322 (2006)

4. Schievano, S., Migliavacca, F., Coats, S., Khambadkone, L., Carminati, M., Wilson, N., Deanfield, J., Bonhoeffer, P., Taylor, A.: Percutaneous pulmonary valve implantation based on rapid prototyping of right ventricular outflow tract and pulmonary trunk from mr data. Radiology 242(2), 490-499 (2007)

5. Schievano, S., Coats, L., Migliavacca, F., Norman, W., Frigiola, A., Deanfield, J., Bonhoeffer, P., Taylor, A.: Variations in right ventricular outflow tract morphology following repair of congenital heart disease: Implications for percutaneous pulmonary valve implantation. Journal of Cardiovascular Magnetic Resonance 9(4), 687-695 (2007)

6. Bonhoeffer, P., Boudjemline, S.A., Qureshi, Y., Bidois, J.L., Iserin, L., Acar, P., Merckx, J., Kachaner, J., Sidi, D.: Percutaneous insertion of the pulmonary valve. Journal of the American College of Cardiology 39(10), 1664-1669 (in press, 2002)

7. Piegl, L., Tiller, W.: The NURBS book. Springer, London (1995) 
8. Zheng, Y., Barbu, A., et al.: Fast automatic heart chamber segmentation from 3d ct data using marginal space learning and steerable features. In: ICCV (2007)

9. Tu, Z.: Probabilistic boosting-tree: Learning discriminativemethods for classification, recognition, and clustering. In: ICCV 2005, pp. 1589-1596 (2005)

10. Zheng, Y., Barbu, A., Georgescu, B., Scheuering, M., Comaniciu, D.: Four-chamber heart modeling and automatic segmentation for 3 -d cardiac ct volumes using marginal space learning and steerable features. IEEE Transactions on Medical Imaging 27(11), 1668-1681 (2008)

11. Cootes, T.F., Taylor, C.J., Cooper, D.H., Graham, J.: Active shape models-their training and application. Computer Vision and Image Understanding 61(1), 38-59 (1995)

12. Yang, L., Georgescu, B., Zheng, Y., Meer, P., Comaniciu, D.: 3d ultrasound tracking of the left ventricle using one-step forward prediction and data fusion of collaborative trackers. In: CVPR (2008)

13. Nollen, G., Schijndel, K., Timmermans, J., Groenink, M., Barentsz, J., Wall, E., Stoker, J., Mulder, B.: Pulmonary artery root dilatation in marfan syndrome: quantitative assessment of an unknown criterion. Heart 87(5), 470-471 (2002)

14. Ionasec, I.I., Tsymbal, A., Vitanovski, D., Georgescu, B., Zhou, S., Navab, N., Comaniciu, D.: Shape-based diagnosis of the aortic valve. In: SPIE Medical Imaging, Orlando, USA (February 2009) 